\title{
Aplicação da Health 4.0 no direcionamento de consultas especializadas em um hospital
}

\author{
Applying Health 4.0 in directing specialized \\ consultations in a hospital
}

\author{
Alexandre Silva Souza ${ }^{1}$ \\ Irapuan Glória Júnior²
}

\begin{abstract}
Resumo
Em uma sociedade tecnicista os dados podem exercer um efeito poderoso da vida pessoas, e muitas vezes isso ocorre de forma discreta. A Health 4.0 tenta fazer uso desses dados para efetuar avanços na saúde e bem-estar das pessoas, como o uso de informações médicas coletadas diariamente para melhorar os processos hospitalares. A pesquisa utilizou a metodologia de estudo de caso único para a construção de uma solução computacional. O resultado é um totem que apresenta a especialidade mais adequada para um paciente ao chegar em um hospital, baseado na indicação de sua enfermidade, seu histórico médico e nas especialidades disponíveis para auxiliar no processo de triagem do hospital. A contribuição para a prática é a apresentação de um modelo que poderá servir para os gestores que necessitam de soluções similares ao contexto estabelecido. A contribuição teórica é a de demonstrar outras aplicações para os conceitos da Health 4.0
\end{abstract}

Palavras-chave: Health 4.0; Solução Computacional; Processo de Triagem.

\begin{abstract}
In a technicist society data can exert a powerful effect on people's lives, and often this occurs discreetly. Health 4.0 attempts to make use of this data to make advances in people's health and wellbeing such as the use of medical information collected daily to improve hospital processes. The research used the unique case study methodology for the construction of a computational solution. The

\footnotetext{
Instituto Federal de São Paulo - IFSP Capivari, São Paulo, Brasil. alexandres.souza@hotmail.com

Instituto Federal de São Paulo - IFSP Capivari, São Paulo, Brasil.ORCID: https://orcid.org/0000-0003-2973-3470 | ijunior@ndsgn.com.br
}

\section{Como citar:}

Souza, A. S., \& Glória Júnior, I. (2020). Aplicação da Health 4.0 no direcionamento de consultas especializadas em um hospital. Revista Inovação, Projetos e Tecnologias, 8(1), 1-12. https://doi.org/10.5585/iptec.v8i1.14339 
result is a totem that presents the most suitable specialty for a patient upon arrival in a hospital, based on the indication of their illness, their medical history and the specialties available to aid in the hospital's screening process. The contribution to the practice is the presentation of a model that may be useful for managers who need solutions similar to the established context. The theoretical contribution is to demonstrate other applications for Health 4.0 concepts.

Keywords: Health 4.0; Computational Solution; Screening Process.

\section{Introdução}

As empresas começam a experimentar a quarta etapa da revolução industrial chamada de Indústria 4.0 (Ind4) que tem como objetivo impactar significativamente as indústrias de manufatura por meio da ideia de conectar e integrar maquinas, pessoas e sistemas de informação com o objetivo de alcançar um melhor controle no ciclo de vida dos produtos e atender as necessidades dos clientes de forma individual (Despeisse et al., 2016).

A Ind4 provocou desdobramentos de seus conceitos por meio das mais diversas áreas como a comunicação, a saúde (Sannino, Falco, \& Pietro, 2019), o transporte (Poli, Saviani, \& Glória Júnior, 2018), dentre outros. No campo da saúde emergiu o conceito de Health 4.0 que contempla a produção de conhecimento medicinal para assistências médicas personalizadas, tanto a pacientes quanto aos profissionais da área da saúde e o bem estar das pessoas (Labuda, Lepa, Labuda, \& Kozak, 2017).

A constante geração de informações é uma característica da saúde no qual auxilia na gestão do tratamento de doenças, noções das condições dos pacientes e até mesmo na educação da medicina, em contrapartida, sua carência de informações em determinadas situações podem acarretar em complicação num quadro médico ou até mesmo resultar em uma mortalidade (Monteiro et al., 2018).

A Empresa-A requisitou o desenvolvimento de um totem que pudesse coletar informações específicas do paciente, identificá-lo e direcioná-lo para o profissional mais adequado. Em virtude dessa requisição esse artigo responde a seguinte questão de pesquisa: "Como um totem pode auxiliar no direcionamento do profissional mais adequado a situação atual?”, desta forma apresenta uma solução computacional de um totem com o uso da Health 4.0 e com o uso de outras tecnologias disponíveis para realizar os direcionamentos para consultas médicas em um centro hospitalar de forma mais eficiente, com base na situação atual e do seu histórico médico, com o intuito de direcionar o atendimento para os profissionais mais aptos para efetuar a consulta. Os objetivos são: (1) Realizar o levantamento de requisitos; e (2) Apresentar uma solução computacional que contemple os requisitos identificados.

Este trabalho está dividido em seções, sendo na Seção 1 a introdução ao tema. Na seção 2 há o referencial teórico nos eixos de gestão de projetos e Health 4.0. A metodologia utilizada foi apresentada na Seção 3. O desenvolvimento da solução está na Seção 4. Na seção 5 estão as conclusões, contribuições para a prática e teoria, além de futuros trabalhos.

\section{Referencial Teórico}

\subsection{Gestão de projetos}

Um projeto consiste em um conjunto de ações para produzir um produto, serviço ou resultado específico (PMI, 2017), sendo planejado dentro de um orçamento e um cronograma e que o início e seu termino são programados (Jiang \& Fernandez, 2018).

No ramo de desenvolvimento de sistemas, o produto a ser elaborado é um sistema computacional, caracterizado em seus requisitos e em suas limitações, que fornecem suporte ou 
automatizam uma determinada tarefa (Pressman, 2011).

O processo de levantamento de requisitos é de grande importância para um projeto bem-sucedido, pois são neles que são apresentados os parâmetros que o sistema deve possuir, como: objetivos, funções, restrições, propriedades (Sommerville, 2011). Esses parâmetros são definidos em acordo com a equipe do projeto e as partes interessadas no projeto, os Stakeholders (PMI, 2017).

O sistema computacional e seus requisitos são expressados por meio de diagramas, cuja finalidade é esclarecer o escopo do projeto ( Jiang \& Fernandez, 2018). Na produção de sistemas a Unified Modeling Language (UML) é uma linguagem de notação utilizada para o desenvolvimento desses diagramas, sendo os principais: (1) Diagrama de Caso de Uso, que mostra uma visão geral das funções do sistema e o (2) Diagrama de Atividade, que exibe o fluxo que as atividades serão produzidas.

\subsection{Health 4.0}

A Ind4 começa a ser discutida nas empresas e poderá se tornar uma tendência entre as indústrias, impactando na forma do gerenciamento da informação e na tomada de decisões (Badri, Boudreau-Trudel, \& Souissi, 2018), desta forma gerando um transbordamento tecnológico para as mais diversas áreas, como na área da saúde como Health 4.0.

O conceito de Health 4.0. (H4) é aproveitar de qualquer tipo de dado médico para o avanço da medicina, no qual permitirá uma melhor avaliação de doenças e previsão de terapia para muitas outras doenças (Andrade et al., 2019; Collins \& Varmus, 2015). A H4 passa por um período de consolidação no mercado, com novas pesquisas, diferentemente da Ind4 que possui fundamentos mais estabelecidos, no qual interliga diversos serviços tecnólogos para a produção de informação e automatização de processos, como: artificial intelligence e a Maching Learning (Labuda et al., 2017).

O ramo de Artificial Intelligence (AI) está apresentando diversos avanços em diversas áreas, como assistente pessoal eletrônica como a Siri da Apple (https://www.apple.com/br/siri/) ou a Alexa da Amazon (https://www.amazon.com), veículos em a necessidade de um motorista, esses e outros projetos em que essas tecnologias podem impactaram a maneira de como a sociedade se comporta (Fogel \& Kvedar, 2018).

Outro aspecto da AI é a utilização de técnicas de Maching Learning (ML) que analisam dados estruturados, como um banco de dados ou um arquivo XML, para tentar relaciona-los ou inferir probabilidade, e os métodos de processamento de linguagem que consigam extrair informações de dados não estruturados e transforma-los em dados estruturados que poderão ser interpretados e utiliza de algoritmos para constatar padrões de dados e assim aprender com eles de forma cíclica (Jiang et al., 2017).

Existem dois principais tipos de categorias referende a aprendizagem de máquina: a supervisionada, ocorrida quando o algoritmo inicialmente recebe uma certa quantidade de padrões de dados como uma carga inicial, e a não supervisionada, que pelo contrário da supervisionada, precisa encontrar e aprender os padrões dos dados sem o auxílio de nenhuma informação prévia (Wahl, Cossy-Gantner, Germann, \& Schwalbe, 2018).

\section{Metodologia}

Essa pesquisa possui natureza qualitativa (Martins \& Theóphilo, 2009), utilizou a metodologia de Estudo de Casos Único (Yin, 2015) e a coleta de dados foi realizada junto aos usuários-chave da Empresa-A. A unidade de análise foi o projeto de direcionamento de consultas a pacientes em um centro hospitalar por meio de um totem inteligente. 


\subsection{Procedimentos metodológicos}

Os procedimentos para a realização da pesquisa foram:

Passo1: Levantar os Requisitos. Nessa etapa foram obtidos os requisitos funcionais da solução computacional por meio de entrevistas junto aos usuários-chaves;

Passo2: Analisar e Projetar o Sistema. A solução foi modelada e documentada por meio de diagramas da UML comumente utilizada nas empresas de desenvolvimento (Pressman, 2011; Sommerville, 2011);

Passo3: Construir o Protótipo do Sistema. Apresentação as interfaces gráficas do software com suas devidas descrições;

Passo4: Avaliar o Sistema. Baseado nos requisitos, uma tabela foi exibida contendo as relações entre os requisitos e os processos que os abrangem.

\section{Resultados obtidos e análise}

\subsection{Levantamento de requisitos}

O totem para o direcionamento de consultas possui o intuito de auxiliar no processo de triagem de um centro hospitalar encaminhando pacientes para médicos especializados no prédio, que seguiu os requisitos identificados junto aos usuários-chaves da Empresa-A e estão apresentados na Tabela 1.

\section{Tabela 1 - Requisitos Funcionais}

\begin{tabular}{lll}
\hline$\#$ & Requisito Funcional & Descrição \\
\hline RF01 & Gerenciar Paciente & Cadastrar, editar e excluir conta e efetuar o login no totem inteligente. \\
\hline RF02 & Ler Cartão do Paciente & Realizar leitura do cartão do paciente que contém as informações do paciente. \\
\hline RF03 & Ler Biometria do Paciente & Realizar leitura da biometria do paciente \\
\hline RF04 & Coletar Dados Hospitalares & Realizar coleta dos dados medidos do paciente através do oxímetro. \\
\hline RF05 & Apontar Região com Dor & Capturar a área indicada pelo paciente que apresenta dor. \\
\hline RF06 & Indicar a Intensidade da Dor & $\begin{array}{l}\text { Informar a intensidade da dor através de uma escala: suportável, moderada, intensa, } \\
\text { insuportável. }\end{array}$ \\
\hline RF07 & $\begin{array}{l}\text { Consultar Histórico do } \\
\text { Paciente }\end{array}$ & Pesquisar histórico médico do paciente para auxílio do direcionamento \\
\hline RF08 & Identificar Especialidade & $\begin{array}{l}\text { Apontar a especialidade médica através dos resultados obtidos dos exames e da } \\
\text { região indicada no avatar. }\end{array}$ \\
\hline RF09 & $\begin{array}{l}\text { Indicar o caminho a ser } \\
\text { seguido }\end{array}$ & \begin{tabular}{l} 
Dirigir o paciente para uma das faixas no solo que o levará especialidade necessária \\
\hline
\end{tabular} \\
\hline
\end{tabular}

Fonte: Autores.

Os requisitos levantados descreveram o funcionamento que solução tem deverá apresentar, em que processo de funcionamento do totem é iniciado no cadastramento dos pacientes (RF01) em uma aplicação WEB para o acesso ao totem. $\mathrm{O}$ acesso ao totem será realizado por meio de uma leitura do 
cartão hospitalar (RF02) ou a biometria do paciente (RF03) para a leitura dos dados pessoais juntamente com o histórico médico do paciente.

Após a coleta dos dados do paciente, é realizada a obtenção dos sinais vitais do paciente (RF04) por meio de um oxímetro, que é um equipamento de monitoramento de oxigenação sanguínea e pulsação corpórea, que foi instalado no totem.

Tendo a coleta dos sinais vitais concluída, o paciente precisará indicar a região do corpo afetada pela dor (RF05) por meio em avatar exibido na tela touchscreen do totem e classificar a intensidade da dor que o incômodo se encontra (RF06).

Na próxima etapa a aplicação instalado no totem irá buscar uma relação entre a região indicada pelo paciente e histórico médico (RF07) para identificar qual especialidade (RF08) o paciente precisará se consultar e o seu caminho a ser percorrido (RF09).

\subsection{Analisar e projetar o sistema}

O projeto consiste em posicionar o totem próximo a entrada do Pronto Atendimento para facilitar o acesso por parte dos pacientes, conforme a Figura 1. Finalizado a interação com o totem, após processo de indicação da intensidade do incômodo, o sistema indicará qual especialidade o paciente será consultado e exibirá qual faixa deve ser percorrida.

A diagramação do sistema foi realizada por meio da UML, onde constituirá representado com os diagramas de caso de uso e atividade. No Diagrama de Uso Gerenciar Conta Paciente, apresentado na Figura 2, possui como proposta apresentar os processos que podem ser realizados no gerenciamento do Paciente.

O cadastramento dos pacientes, será realizado em uma aplicação WEB no qual um recepcionista irá inserir dos dados pessoais dos pacientes (P01) que necessitaram ser validados antes de serem inseridos na base de dados (P02). Caso as informações inseridas forem válidas a criação da conta do paciente no centro hospital será realizada (P03), do contrário será exibido uma mensagem de erro na abertura da conta (P04).

Se o paciente já se dispor de uma conta ativa, ao buscar os dados do mesmo, será exibida as opções de manutenção da conta (P05) em que ao selecionar a opção desejada (P06) o usuário poderá excluir a conta desse paciente (P07) ou modificar as informações do cadastro do paciente que precisarão passar por uma nova etapa de validação dos dados (P08) a fim que esses novos dados sejam atualizados na base (P09). 


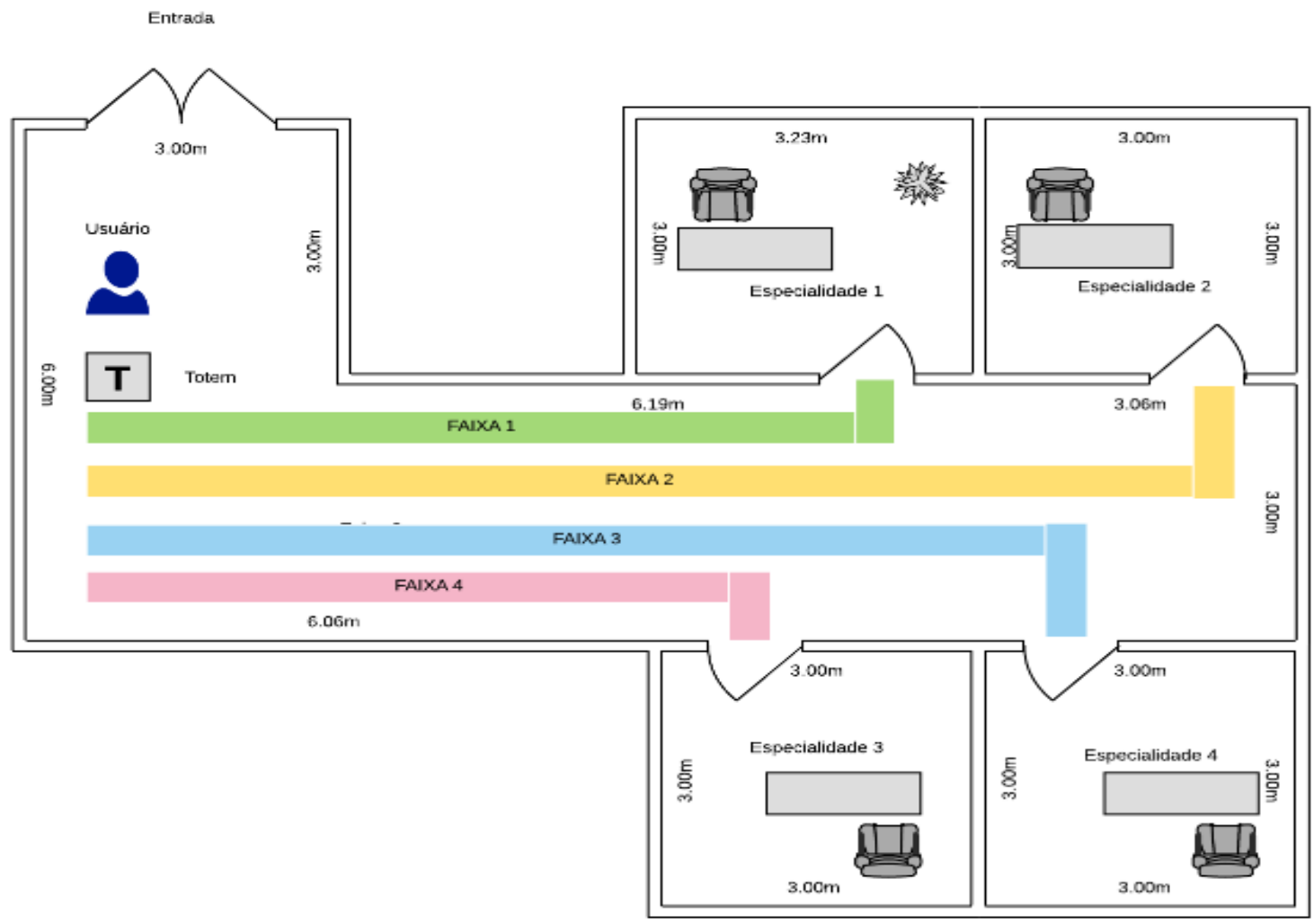

Figura 1 - Localização do Totem

Fonte: Autores.

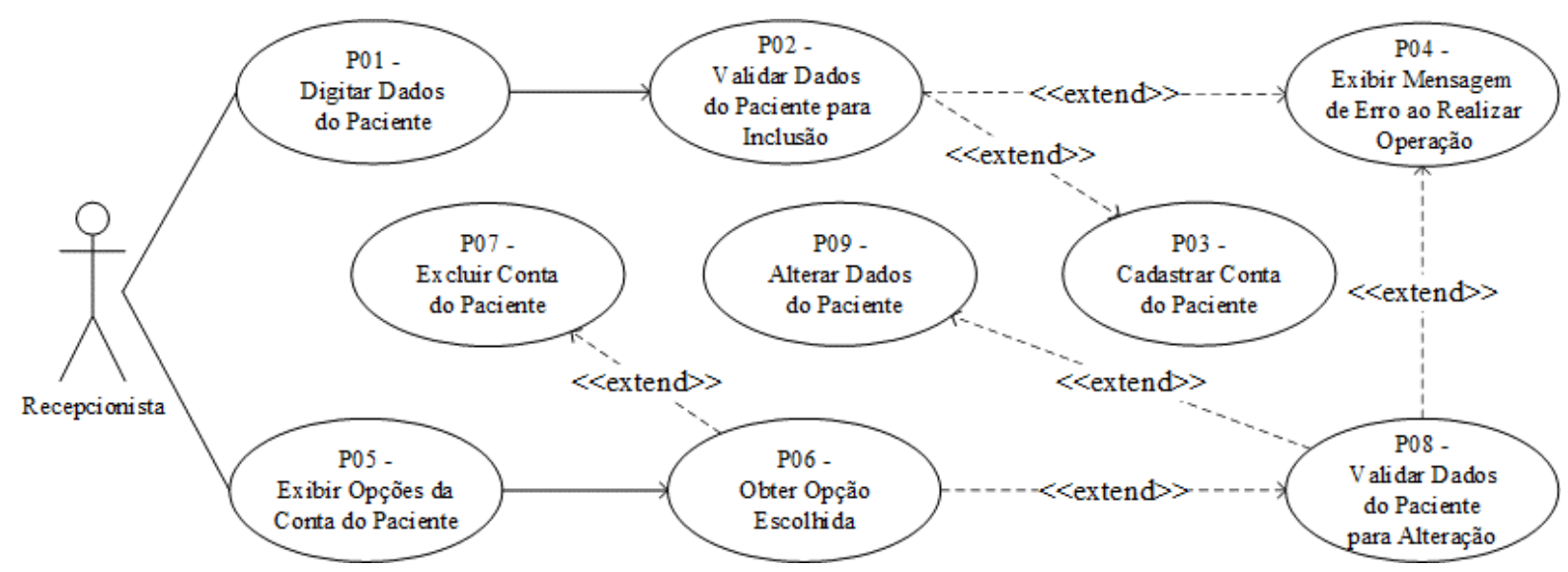

Figura 2 - Gerenciar Conta Paciente

Fonte: Autores.

O diagrama de Caso de Uso Interação com o Totem retratado na Figura 3 tem como proposta apresentar os processos que podem ser realizados pelo paciente por meio do totem. 


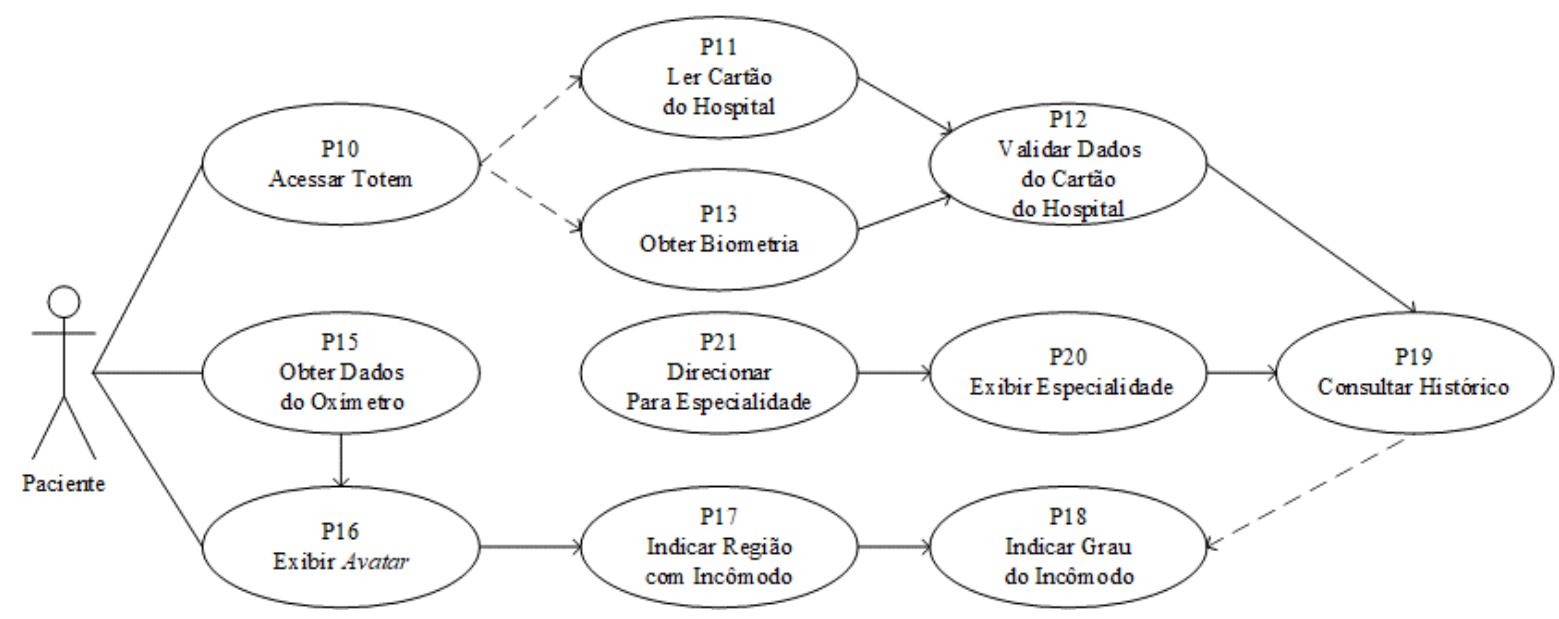

Figura 3 - Interação com o Totem

Fonte: Autores

Para interagir com o totem (P10) faz-se necessário o paciente necessita realizar um processo de identificação meio de seu cartão do hospital (P11) ou por sua biometria (P13) no qual serão validados para obter a permissão de entrada (P12 e P14).

Tendo acessado o totem com êxito, a próxima etapa é realizar a captura dos sinais vitais do paciente que será executado por meio de um oxímetro (P15), assim, após a obtenção dos dados do oxímetro, será exibido na tela um atavar correspondente as informações do paciente (sexo, altura, período de gestação, dentre outras informações) no qual poderá ser apontado a região que se apresenta com incômodo (P16) e grau desse desconforto (P17).

A partir disso o totem buscará no histórico médico do paciente a fim de traçar alguma relação com o estado atual (P18). Após a consulta no histórico médico, o totem exibirá a especialidade que o paciente realizará sua consulta (P19) e qual caminho ele deve seguir (P20).

O diagrama de atividade Interação com o Totem, mostrado na Figura 4, tem como proposta ilustrar o fluxo da interação do paciente com o totem. O bloco 1 corresponde a primeira interatividade com o paciente e, dependendo se possui cartão do convênio (Bloco 2) ou não (Bloco 3) será identificado. Em seguida será realizada a leitura e indicação de seu estado atual (Blocos 4 a 6), o sistema irá consultar seu histórico (Bloco 7) e apresentar o direcionamento (Blocos 8 e 9). 


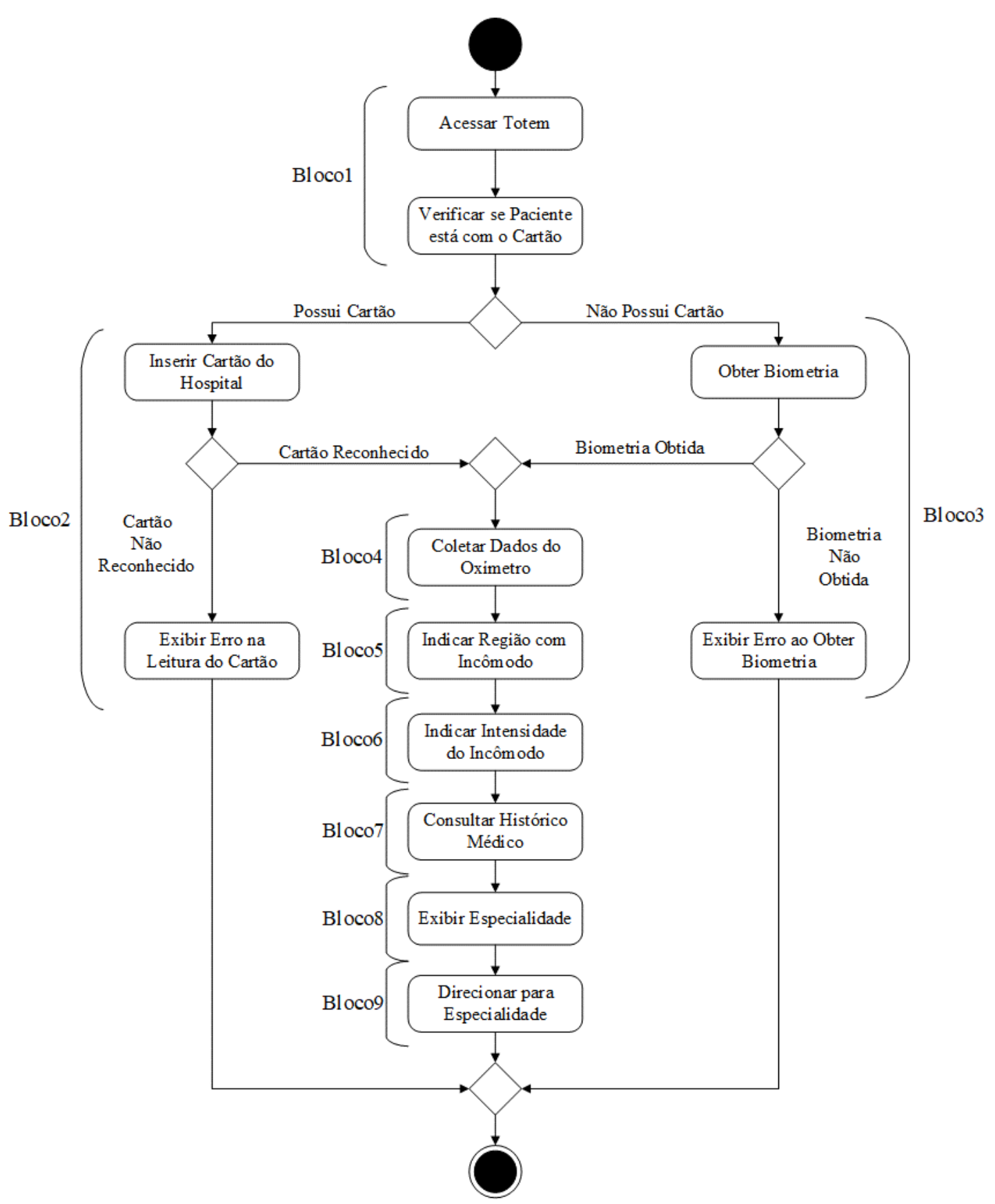

Figura 4 - Fluxo da Interação com o Totem

Fonte: Autores.

\subsection{Construir o protótipo do sistema}

$\mathrm{O}$ artefato de pesquisa será um totem inteligente cujo objetivo é se comportar como um appliance visando uma interação com o usuário da forma mais simples possível e que seja executado sob uma interface com o intuito de diminuir a atenção com alguns fatores como sua instalação, a administração e manutenção (Gartner, 2019).

O totem será composto por um leitor de cartão, um leitor de biometria, um oxímetro e uma tela touch screen, conforme ilustrado na Figura 5. 


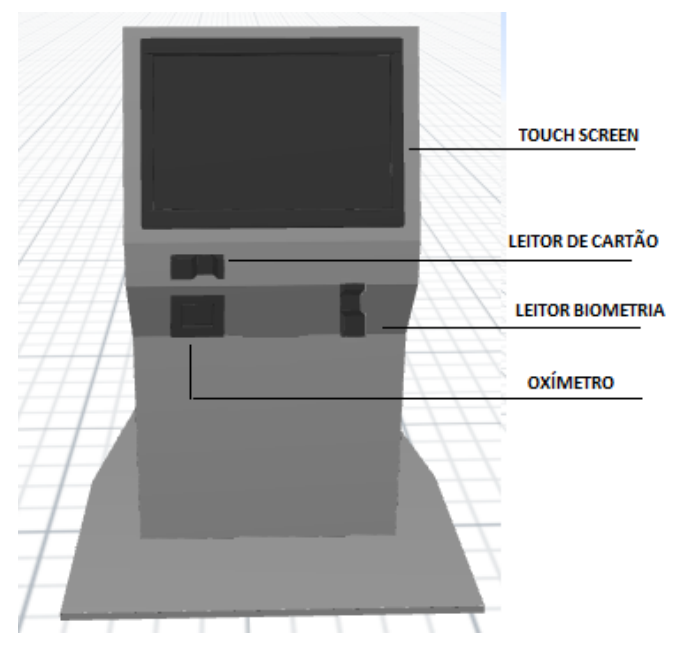

Figura 5 - Protótipos do Totem

Fonte: Autores.

Tendo acessado o totem por meio de seu cartão/biometria e após a coleta dos dados por meio do oxímetro, será exibido um avatar que corresponde as características físicas do paciente: sexo, cor da pele, dentre outras características, conforme a Figura 6.

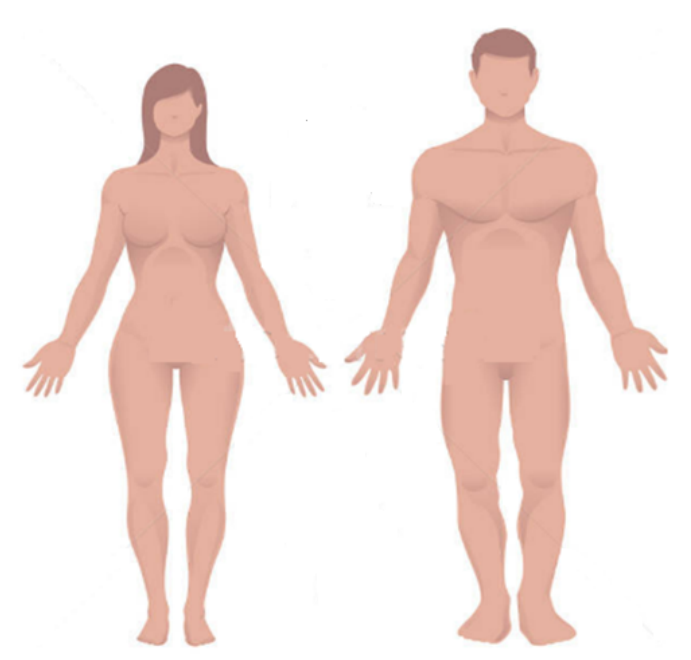

Figura 6 - Avatar para Indicação do Incômodo

Fonte: Autores.

$\mathrm{Na}$ tela de touch screen, o paciente poderá indicar a região que se apresenta o incômodo e classificar seu estado por meio de uma escala likert (Martins \& Theóphilo, 2009) de medição de dor que começa em um incômodo suportável, moderado, intenso e por fim insuportável.

Para exemplificar o uso na Figura 7 o avatar do sexo masculino é apresentado com a região do peito localizado no lado direito marcado e as opções de níveis de dor à ser escolhida pelo paciente ou acompanhante. 


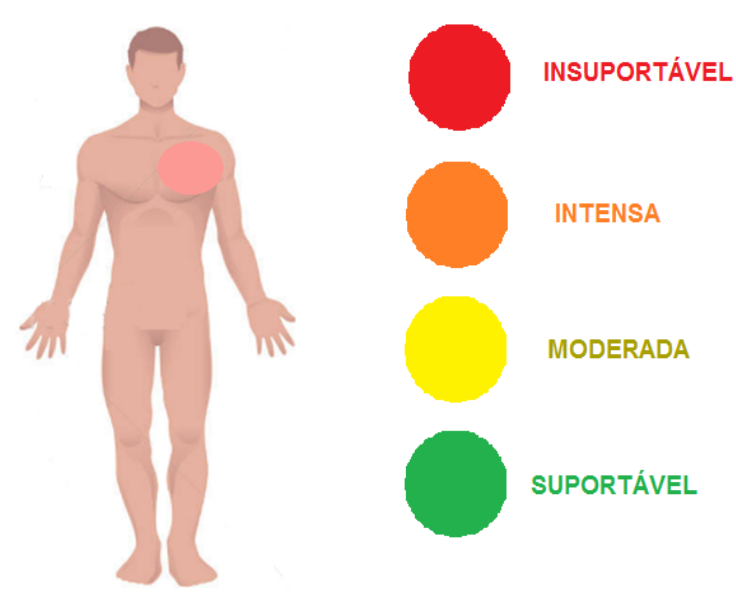

Figura 7 - Avatar com região marcada

Fonte: Autores.

\subsection{Avaliar o sistema}

A avaliação o protótipo, apresentada na Tabela 2, apresenta a relação entre os requisitos funcionais apresentados na etapa de construção do framework conceitual com os diagramas elaborado na etapa de análise e projeto do sistema por meio dos diagramas de caso de uso, o diagrama de atividade.

Tabela 2 - Relação Entre os Requisitos Funcionais e os Diagramas

\begin{tabular}{|c|c|c|c|}
\hline$\#$ & Requisito Funcional & $\begin{array}{l}\text { Diagrama de } \\
\text { Caso de Uso }\end{array}$ & $\begin{array}{l}\text { Diagrama de } \\
\text { Atividades }\end{array}$ \\
\hline RF01 & Gerenciar Conta Paciente & $\begin{array}{l}\text { P01 - Digitar Dados do Paciente } \\
\text { P02 - Validar Dados do Paciente para Inclusão } \\
\text { P03 - Validar Dados do Paciente } \\
\text { P04 - Cadastrar Conta do Paciente } \\
\text { P05 - Exibir Opções da Conta do Paciente } \\
\text { P06 - Obter Opção Escolhida } \\
\text { P07 - Excluir Conta do Paciente } \\
\text { P08 - Validar Dados do Paciente para Alteração } \\
\text { P09 - Alterar Dados do Paciente }\end{array}$ & Bloco1 \\
\hline RF02 & Ler Cartão do Paciente & $\begin{array}{l}\text { P10 - Acessar Totem } \\
\text { P11 - Ler Cartão do Hospital } \\
\text { P12 - Validar Dados do Cartão do Hospital }\end{array}$ & Bloco2 \\
\hline RF03 & Ler Biometria do Paciente & $\begin{array}{l}\text { P10 - Acessar Totem } \\
\text { P13 - Obter Biometria } \\
\text { P14 - Validar Dados da Biometria } \\
\end{array}$ & Bloco3 \\
\hline RF04 & Coletar Dados Hospitalares & P15 - Obter Dados do Oxímetro & Bloco4 \\
\hline RF05 & $\begin{array}{l}\text { Apontar Região com } \\
\text { Incômodo }\end{array}$ & $\begin{array}{l}\text { P16 - Exibir Avatar } \\
\text { P17 - Indicar Região com Incômodo }\end{array}$ & Bloco5 \\
\hline RF06 & $\begin{array}{l}\text { Indicar a Intensidade do } \\
\text { Incômodo }\end{array}$ & $\begin{array}{l}\text { P16 - Exibir Avatar } \\
\text { P17 - Indicar Grau do Incômodo }\end{array}$ & Bloco6 \\
\hline RF07 & $\begin{array}{l}\text { Consultar Histórico do } \\
\text { Paciente }\end{array}$ & P19 - Consultar Histórico & Bloco7 \\
\hline RF08 & Identificar Especialidade & P20 - Exibir Especialidade & Bloco 8 \\
\hline RF09 & $\begin{array}{l}\text { Indicar o caminho a ser } \\
\text { seguido }\end{array}$ & P21 - Direcionar para Especialidade & Bloco 9 \\
\hline
\end{tabular}

Fonte: Autores 


\section{Considerações finais}

O advento da Indústria 4.0 produziu o transbordamento tecnológico para outras áreas, como a da saúde gerando a Health 4.0, que depende de informações para poder gerar decisões para o bemestar dos pacientes e as tecnologias disponíveis no mercado podem corroborar para sua implantação.

Esse trabalho resulta em uma solução computacional em forma de um protótipo conceitual de um totem inteligente que possui a finalidade de proporcionar maior velocidade no processo de triagem e poderá auxiliar na acurácia dos diagnósticos realizados pelos médicos.

A limitação deste trabalho é a de que o totem ainda está em processo de prototipação pela Empresa-A. A contribuição para a prática é a apresentação de um modelo que poderá servir para os gestores que necessitam de soluções similares ao contexto estabelecido. A contribuição teórica é a de demonstrar outras aplicações para os conceitos da Health 4.0. Como futuros trabalhos está a criação da conectividade entre diversos centros hospitalares por meio dos totens inteligentes e a criação de um Big Data com as informações coletadas pelo totem com o intuito de obter indicações de epidemias.

\section{Referências}

Andrade, N., Costa Neto, P. L. O., Torres, J. G. M., Glória Júnior, I., Scheidt, C. G., \& Gazel, W. (2019). EHealth: A framework proposal for interoperability and health data sharing. A Brazilian Case. IFIP International Conference on Advances in Production Management Systems, 1, 544-551. Austin: Springer.

Badri, A., Boudreau-Trudel, B., \& Souissi, A. S. (2018). Occupational health and safety in the industry 4.0 era: A cause for major concern? Safety Science, 109, 403-411. https://doi.org/10.1016/j.ssci.2018.06.012

Collins, F. S., \& Varmus, H. (2015). A New Initiative on Precision Medicine. New England Journal of Medicine, 372(9), 793-795. https://doi.org/10.1056/NEJMp1500523

Despeisse, M., Davé, A., Litos, L., Roberts, S., Ball, P., \& Evans, S. (2016). A Collection of Tools for Factory Eco-efficiency. Procedia CIRP, 40, 542-546. https://doi.org/10.1016/j.procir.2016.01.130

Fogel, A. L., \& Kvedar, J. C. (2018). Artificial intelligence powers digital medicine. Npj Digital Medicine, 1(1). https://doi.org/10.1038/s41746-017-0012-2

Gartner. (2019). Computing Appliance. Recuperado 11 de junho de 2019, de https://www.gartner.com/itglossary/computing-appliance/

Jiang, F., Jiang, Y., Zhi, H., Dong, Y., Li, H., Ma, S., .. Wang, Y. (2017). Artificial intelligence in healthcare: Past, present and future. Stroke and Vascular Neurology, 2(4), 230-243. https://doi.org/10.1136/svn2017-000101

Jiang, J., \& Fernandez, W. (2018). From Project Management to Program Management: An Invitation to Investigate Programs Where IT Plays a Significant Role. Journal of the Association for Information Systems, 19(1), 40-57. https://doi.org/10.17705/1jais.00480

Labuda, N., Lepa, T., Labuda, M., \& Kozak, K. (2017). Medical 4.0: Medical Data Ready for Deep and Machine Learning. Journal of Bioanalysis \& Biomedicine, 09(06). https://doi.org/10.4172/1948593X.1000194

Martins, G. de A., \& Theóphilo, C. R. (2009). Metodologia da Investigação Científica Para Ciências Sociais Aplicadas. São Paulo: Atlas.

Monteiro, A. C. B., França, R. P., Estrela, V. V., Iano, Y., Khelassi, A., \& Razmjooy, N. (2018). Health 4.0: Applications, Management, Technologies and Review. Medical Technologies Journal, 2(4), 15. 
PMI. (2017). Project Management Body of Knowledge Guide. Pennsylvania: Four Campus Boulevard.

Poli, G. A., Saviani, T. N., \& Glória Júnior, I. (2018). Logistics 4.0: A Systematic Review. 9(2), $32-47$.

Pressman, R. (2011). Engenharia de Software (6 ${ }^{\circ}$ ed). São Paulo: McGraw-Hill.

Sannino, G., Falco, I. D., \& Pietro, G. D. (2019). A Continuous Noninvasive Arterial Pressure (CNAP) Approach for Health 4.0 Systems. IEEE Transactions on Industrial Informatics, 15(1), 498-506. https://doi.org/10.1109/TII.2018.2832081

Sommerville, I. (2011). Engenharia de Software ( $9^{\circ}$ ed). São Paulo: Pearson Education.

Wahl, B., Cossy-Gantner, A., Germann, S., \& Schwalbe, N. R. (2018). Artificial intelligence (AI) and global health: How can AI contribute to health in resource-poor settings? BMJ Global Health, 3(4), e000798. https://doi.org/10.1136/bmjgh-2018-000798

Yin, R. K. (2015). Estudo de caso: planejamento e métodos. Porto Alegre: Bookman. 